\title{
HYPERINVARIANT SUBSPACES OF OPERATORS ON HILBERT SPACES
}

\author{
ŠTEFAN DRAHOVSKÝ \\ Faculty of Mathematics and Physics, Comenius University \\ Mlynská dolina, 84215 Bratislava, Slovakia \\ MICHAL ZAJAC \\ Department of Mathematics, Faculty of Electrical Engineering, Slovak Technical University \\ 81219 Bratislava, Slovakia \\ E-mail: zajac@kmat.elf.stuba.cs
}

1. Introduction. Let $H$ be a complex separable Hilbert space, $\mathcal{B}(H)$ the algebra of all continuous linear operators on $H$ and $T \in \mathcal{B}(H)$. We denote by $\{T\}^{\prime}$ the commutant of $T\left(X \in\{T\}^{\prime}\right.$ if and only if $\left.X T=T X\right)$ and by $\{T\}^{\prime \prime}=\bigcap\left\{\{X\}^{\prime}\right.$ : $X T=T X\}$ the bi-commutant of $T$. A contraction means an operator $T \in \mathcal{B}(H)$ with norm $\|T\| \leq 1$. By a subspace we always mean a closed linear subspace. A subspace $L \subset H$ is called invariant for $T \in \mathcal{B}(H)$ if $T L \subset L$, and hyperinvariant (bi-invariant) for $T$ if it is invariant for every $X \in\{T\}^{\prime}\left(X \in\{T\}^{\prime \prime}\right)$. If $\mathcal{A} \subset \mathcal{B}(H)$ then $\operatorname{Alg} \mathcal{A}$ denotes the smallest weakly closed subalgebra of $\mathcal{B}(H)$ containing $\mathcal{A}$ and the identity $I$, and Lat $\mathcal{A}$ denotes the set of all subspaces invariant for each $A \in \mathcal{A}$. The set Lat $\mathcal{A}$ (with the operations $\cap$ and $\vee$ of intersection and of forming the closed linear span, respectively) is a complete lattice. If $\mathcal{L}$ is a set of subspaces of $H$, then $\operatorname{Alg} \mathcal{L}=\{T \in \mathcal{B}(H): \mathcal{L} \subset$ Lat $T\}$. The significance of these notions for the structure of an operator is obvious, e.g. from [34, Theorems 2.1 and 2.2]. In this paper the following properties of an operator $T \in \mathcal{B}(H)$ are treated:

1991 Mathematics Subject Classification: Primary 47A15.

The paper is in final form and no version of it will be published elsewhere.

We want to express our gratitude to Professor J. Zemánek whose comments led to a better presentation of this paper. 
Definition. Let $T \in \mathcal{B}(H)$. Then

(i) $T$ is said to have the property (L) if $\operatorname{Lat}\{T\}^{\prime}$ is the smallest complete lattice containing all subspaces of the form $\operatorname{ker} S=\{h \in H: S h=0\}$ and $\overline{S H}$ for $S \in\{T\}^{\prime \prime}$,

(ii) $T$ is said to be reflexive if $A \lg T=\operatorname{Alg} \operatorname{Lat} T$,

(iii) $T$ is said to be hyperreflexive if $\{T\}^{\prime}=\operatorname{Alg} \operatorname{Lat}\{T\}^{\prime}$.

The purpose of this paper is to review what is known about the property $(\mathrm{L})$ and hyperreflexivity, and to show the relations between reflexivity and hyperreflexivity.

Remark. Lat $\{T\}^{\prime}$ is often also denoted by Hyplat $T$.

The following lemma is used to reduce the investigation of invariant subspaces and related questions to simpler operators.

Lemma 1. Let the Hilbert space $H$ be the direct sum of two subspaces, $H=$ $H_{1} \oplus H_{2}$. Let $T_{i} \in \mathcal{B}\left(H_{i}\right)(i=1,2)$ and $T=T_{1} \oplus T_{2}$. Consider the following relations:

(1) $\operatorname{Alg}\left(T_{1} \oplus T_{2}\right)=\operatorname{Alg} T_{1} \oplus \operatorname{Alg} T_{2}$

(2) $\operatorname{Lat}\left(T_{1} \oplus T_{2}\right)=\operatorname{Lat} T_{1} \oplus \operatorname{Lat} T_{2}$,

(3) $\operatorname{Alg} \operatorname{Lat}\left(T_{1} \oplus T_{2}\right)=\operatorname{Alg} \operatorname{Lat} T_{1} \oplus \operatorname{Alg} \operatorname{Lat} T_{2}$,

(4) $\left\{T_{1} \oplus T_{2}\right\}^{\prime}=\left\{T_{1}\right\}^{\prime} \oplus\left\{T_{2}\right\}^{\prime}$,

(5) $\operatorname{Lat}\left\{T_{1} \oplus T_{2}\right\}^{\prime}=\operatorname{Lat}\left\{T_{1}\right\}^{\prime} \oplus \operatorname{Lat}\left\{T_{2}\right\}^{\prime}$

(6) $\operatorname{Alg} \operatorname{Lat}\left\{T_{1} \oplus T_{2}\right\}^{\prime}=\operatorname{Alg} \operatorname{Lat}\left\{T_{1}\right\}^{\prime} \oplus \operatorname{Alg} \operatorname{Lat}\left\{T_{2}\right\}^{\prime}$,

(7) $\left\{T_{1} \oplus T_{2}\right\}^{\prime \prime}=\left\{T_{1}\right\}^{\prime \prime} \oplus\left\{T_{2}\right\}^{\prime \prime}$,

(8) $\operatorname{Lat}\left\{T_{1} \oplus T_{2}\right\}^{\prime \prime}=\operatorname{Lat}\left\{T_{1}\right\}^{\prime \prime} \oplus \operatorname{Lat}\left\{T_{2}\right\}^{\prime \prime}$,

(9) $\operatorname{Alg} \operatorname{Lat}\left\{T_{1} \oplus T_{2}\right\}^{\prime \prime}=\operatorname{Alg} \operatorname{Lat}\left\{T_{1}\right\}^{\prime \prime} \oplus \operatorname{Alg} \operatorname{Lat}\left\{T_{2}\right\}^{\prime \prime}$.

Then the following implications hold:

$$
(1) \Rightarrow(2) \Leftrightarrow(3) \Rightarrow(4) \Leftrightarrow(5) \Leftrightarrow(6) \Leftrightarrow(7) \Rightarrow(8) \Leftrightarrow(9) .
$$

Remarks. The proof of this lemma is simple and can be found e.g. in [8], [40]. (1) (and so all the other relations) is valid e.g. if $\operatorname{dim} H<\infty$ and the minimal polynomials of $T_{1}$ and $T_{2}$ are relatively prime.

If $\operatorname{dim} H=\infty$ and $\|T\| \leq 1$, then (1) holds true if $T_{1}$ is the absolutely continuous part and $T_{2}$ is the singular unitary part of $T$. If $T$ is a contraction of class $C_{0}$ in the sense of [26], then (1) holds if the minimal functions of $T_{1}$ and $T_{2}$ are relatively prime.

2. Operators on finite-dimensional spaces. Let $\operatorname{dim} H<\infty$. Let $T \in$ $\mathcal{B}(H)$ have minimal polynomial

$$
m_{T}(\lambda)=\prod_{i=1}^{n}\left(\lambda-\lambda_{i}\right)^{m_{i}} .
$$

Let $H_{i}=\operatorname{ker}\left(T-\lambda_{i} I\right)^{m_{i}}$. Then the following assertions hold: 
(i) $H_{i} \in \operatorname{Lat}\{T\}^{\prime}$ for $i=1, \ldots, n$;

(ii) $H=H_{1} \oplus \ldots \oplus H_{n}$;

(iii) if $T_{i}=T \mid H_{i}$, then $T=\bigoplus_{i=1}^{n} T_{i}$ and $\operatorname{Alg} T=\bigoplus_{i=1}^{n} \operatorname{Alg} T_{i}$.

The minimal polynomial of $T_{i}$ is $\left(\lambda-\lambda_{i}\right)^{m_{i}}$. All the objects considered in Lemma 1 remain unchanged if we pass from $T_{i}$ to the nilpotent operator $T_{i}-\lambda_{i}$. Therefore most problems concerning invariant subspaces of operators on finitedimensional spaces reduce to the case of a nilpotent operator. Let $N \in \mathcal{B}(H)$ be a nilpotent operator of order $n$ (i.e. $N^{n}=0, N^{n-1} \neq 0$ ). We consider its Jordan form, i.e. we assume that the matrix representation of $N$ is

$$
N=J\left(k_{1}\right) \oplus \ldots \oplus J\left(k_{m}\right), \quad n=k_{1} \geq \ldots \geq k_{m} .
$$

Let the corresponding decomposition of $H$ be

$$
H=H_{1} \oplus \ldots \oplus H_{m} .
$$

Here $J(k)$ is the $k \times k$ Jordan cell (i.e. each entry on the first subdiagonal is 1, and all other entries are 0$)$.

Denote by $e$ the vector $(1,0, \ldots, 0) \in H$. The following theorem puts together results of [7], [10], [12]-[14], and [21].

THEOREM 2. If $N$ is a nilpotent operator given by (I) on the space (II), then the following assertions hold:

(1) $L \in$ Lat $N$ if and only if $L=\operatorname{ker} B$ for an operator $B \in\{N\}^{\prime}$.

(2) $L \in$ Lat $N$ if and only if $L=B H$ for an operator $B \in\{N\}^{\prime}$.

(3) $\operatorname{Alg} N=\{N\}^{\prime \prime}$ and it consists of all polynomials in $N$.

(4) $B \in \operatorname{Alg}$ Lat $N$ if and only if $B=C+D$, where $C \in\{N\}^{\prime \prime}$ and $D$ satisfies the conditions $D H_{1}^{\perp}=\{0\}, D N^{i} e \in \bigvee\left\{N^{k_{2}+i} e, N^{k_{2}+i+1} e, \ldots\right\}$ for $0 \leq i<n$.

(5) $N$ is reflexive if and only if either $k_{1}=k_{2}$ or $k_{1}=k_{2}+1$ (here $k_{2}=0$ if $m=1)$.

(6) Let $A \in \mathcal{B}(H)$ have the block decomposition $A=\left(A_{i j}\right)$ (corresponding to the decomposition (II) of $H$ ). Then

$$
A \in\{N\}^{\prime} \Leftrightarrow\left\{\begin{array}{l}
A_{i i} \in\left\{J\left(k_{i}\right)\right\}^{\prime} \text { for all } i \\
\text { for } i<j, A_{i j}=\left(\begin{array}{c}
0 \\
X
\end{array}\right) \text { with } X \in\left\{J\left(k_{j}\right)\right\}^{\prime} \\
\text { for } i>j, A_{i j}=\left(\begin{array}{ll}
Y & 0
\end{array}\right) \text { with } Y \in\left\{J\left(k_{i}\right)\right\}^{\prime} .
\end{array}\right.
$$

Recall that $\{J(k)\}^{\prime}$ consists of polynomials in $J(k)$ and thus of lower-triangular matrices with equal entries on each subdiagonal $\left(a_{i+1, j+1}=a_{i j}, 1 \leq i \leq k\right.$, $1 \leq j \leq k)$.

(7) $\mathcal{L} \in \operatorname{Lat}\{N\}^{\prime} \Leftrightarrow \mathcal{L}=\bigoplus_{j=1}^{m} \operatorname{ker} J\left(k_{j}\right)^{r_{j}}$ for an m-tuple $r_{1}, \ldots, r_{m}$ of integers with

$$
r_{1} \geq \ldots \geq r_{m} \geq 0, \quad k_{1}-r_{1} \geq \ldots \geq k_{m}-r_{m} \geq 0 .
$$


(8) Let $A \in \mathcal{B}(H)$ have the block decomposition $A=\left(A_{i j}\right)$. Then $A$ belongs to Alg Lat $\{N\}^{\prime}$ if and only if it has the following form:

$$
A_{i j}=\left\{\begin{array}{l}
\text { a lower-triangular matrix if } i=j \\
\left(\begin{array}{l}
0 \\
X
\end{array}\right) \text { with } X \text { lower-triangular if } i<j \\
(Y \quad 0) \text { with } Y \text { lower-triangular if } i>j .
\end{array}\right.
$$

(9) (6) and (8) imply that $N \in \mathcal{B}(H)$ is hyperreflexive if and only if $N=0$.

(10) Lat $N=\operatorname{Lat}\{N\}^{\prime}$ if and only if $m=1$, i.e. the operator $N$ has only one Jordan block.

Remarks. The assertions (1)-(3) hold for arbitrary operators from $\mathcal{B}(H)$. (1) and (2) were proved in [14], the proof of (3) can be found in [12] and [15, Theorem 4.4.19]. From (7) and from Lemma 1 it follows (see [13]) that every $T \in \mathcal{B}(H)$ has the property (L). In [20, Proposition 2] it was shown that every $\mathcal{L} \in \operatorname{Lat}\{T\}^{\prime}$ is the range of an operator $B \in\{T\}^{\prime}$. This can also be proved using (7). (4) and (5) were proved in [10]. Let us point out that (5) means that the reflexivity of $N$ only depends on the dimensions of the largest and second largest Jordan blocks of $N$. (9) and Lemma 1 imply that $T \in \mathcal{B}(H)$ is hyperreflexive if and only if it is similar to a diagonal operator (i.e. all eigenvalues of $T$ have multiplicity 1 ). (10) together with some other equivalent conditions was proved by Ong [21]. In $[25$, Theorem I.3.5] it was proved that if the minimal and characteristic polynomials of an operator $T$ (in a space over an arbitrary field) coincide then $\{T\}^{\prime}$ consists of all polynomials in $T$. A generalization of (5) for an arbitrary scalar field was given in [2].

If $N$ satisfies (9), then $k_{1}=\ldots=k_{m}=1$. Consequently, if $N$ is hyperreflexive, then it is reflexive. Then using Lemma 1 we conclude that hyperreflexivity implies reflexivity for every operator in a finite-dimensional space. In the last section of this paper we show that this is not true in infinite-dimensional Hilbert spaces. The other implication is not even true in finite dimensions, e.g. the operator $\left(\begin{array}{lll}0 & 0 & 0 \\ 1 & 0 & 0 \\ 0 & 0 & 0\end{array}\right)$ is reflexive, but it is not hyperreflexive.

3. Weak contractions. Throughout this section we shall use the terminology and results of Sz.-Nagy and Foias [26]. Let $H_{1}, H_{2}$ be Hilbert spaces. Recall that an operator $X \in \mathcal{B}\left(H_{1}, H_{2}\right)$ is a quasiaffinity if $\operatorname{ker} X=\{0\}$ and $\overline{X H_{1}}=H_{2}$ $\left(\mathcal{B}\left(H_{1}, H_{2}\right)\right.$ is the set of all operators from $H_{1}$ into $\left.H_{2}\right) . T_{2}$ is called a quasiaffine transform of $T_{1}$ if there exists a quasiaffinity $X \in \mathcal{B}\left(H_{1}, H_{2}\right)$ intertwining $T_{1}$ and $T_{2}$, i.e. satisfying $T_{2} X=X T_{1}$. We then write $T_{1} \succ T_{2}$. If both $T_{1} \succ T_{2}$ and $T_{1} \prec T_{2}$, then $T_{1}$ and $T_{2}$ are quasisimilar. These relations play an important role in the theory of functional models of contractions (see e.g. [4], [26]).

It is well known ([26], [38]) that for every contraction $T \in \mathcal{B}(H)$ there exists a unique decomposition of $H$ into the orthogonal direct sum of three subspaces from Lat $T$,

$$
H=H_{1} \oplus H_{2} \oplus H_{3}, \quad T=T_{1} \oplus T_{2} \oplus T_{3},
$$


where $T_{i}=T \mid H_{i}, i=1,2,3$, such that $T_{1}$ is a completely non-unitary (c.n.u.) contraction (i.e. there is no $L \in \operatorname{Lat} T_{1}$ for which $T_{1} \mid L$ is a unitary operator), and $T_{1}$ and $T_{2}$ are absolutely continuous (a.c.) and singular unitary (s.u.) operators (i.e. their spectral measures are absolutely continuous and singular with respect to the Lebesgue measure on the unit circle, respectively). Then $\operatorname{Alg}\left(T_{1} \oplus T_{2} \oplus T_{3}\right)=$ $\operatorname{Alg}\left(T_{1} \oplus T_{2}\right) \oplus \operatorname{Alg}\left(T_{3}\right)$. Using Lemma 1 we may suppose that $T_{3}=0$. Then $T$ is called absolutely continuous. The reason for this terminology is that the minimal unitary dilation ([4], [26]) of $T$ is a.c.

Recall that a contraction $T \in \mathcal{B}(H)$ is a weak contraction if

(i) its spectrum $\sigma(T)$ does not contain the unit disc $D=\{\lambda:|\lambda|<1\}$,

(ii) the operator $I-T^{*} T$ has finite trace.

Since every a.c. weak contraction is similar to a c.n.u. weak contraction [39] it can be assumed that $T$ is c.n.u. when studying invariant and hyperinvariant subspaces of a weak contraction $T$. Moreover, we can use the $C_{0}-C_{11}$ decomposition of $T$ (see [26], [33], [37]):

TheOREM 3. Let $T \in \mathcal{B}(H)$ be a c.n.u. weak contraction. Then there exist $H_{0}, H_{1} \in$ Lat $T$ such that:

(i) $T_{0}=T \mid H_{0} \in C_{0}$ and $T_{1}=T \mid H_{1} \in C_{11}$. ( $C_{0}$ is the class of contractions $T$ for which there exists a bounded analytic function $u$ satisfying $u(T)=0 ; T \in C_{11}$ if for all non-zero $h \in H$, neither $T^{n} h$ nor $T^{* n} h$ converges to 0 .)

(ii) $H_{0} \vee H_{1}=H$ and $H_{0} \cap H_{1}=\{0\}$.

(iii) $H_{0}=\operatorname{ker} m(T), H_{1}=\overline{m(T) H}$, where $m$ is the minimal function of $T_{0}$. Since $m(T) \in\{T\}^{\prime \prime}$, it follows that $H_{0}, H_{1} \in \operatorname{Lat}\{T\}^{\prime}$.

(iv) There exists $S \in\{T\}^{\prime \prime}$ such that $H_{0}=\overline{S H}$ and $H_{1}=\operatorname{ker} S$.

(v) $\operatorname{Lat}\{T\}^{\prime}$ and $\operatorname{Lat}\left\{T_{0}\right\}^{\prime} \oplus \operatorname{Lat}\left\{T_{1}\right\}^{\prime}$ are isomorphic.

If $L \in \operatorname{Lat} T$ and $T$ is a weak contraction the restriction $T \mid L$ need not be weak (its spectrum may contain the whole unit disc). But if $L \in \operatorname{Lat}\{T\}^{\prime}$, then $\sigma(T \mid L) \subset \sigma(T)$ and so $T \mid L$ is a weak contraction. This allows one to show (see [37], [39]) that every weak contraction of class $C_{11}$ has the property (L).

Definition. An operator $T \in \mathcal{B}(H)$ is said to have the property $(\mathrm{P})$ if

$$
(X T=T X \text { and } \operatorname{ker} X=\{0\}) \Rightarrow \overline{X H}=H .
$$

In [35] it was proved that every weak contraction of class $C_{0}$ has the property (L) as a consequence of the fact [3] that every weak $C_{0}$ contraction has the property $(\mathrm{P})$ and every $C_{0}$ contraction having $(\mathrm{P})$ has $(\mathrm{L})$.

Recall that for c.n.u. $C_{11}$ and $C_{0}$ contractions there are some canonical operators (Jordan models) in their quasisimilarity orbits. Denote by $H^{\infty}, H^{2}$ the corresponding Hardy classes of analytic functions and by $H_{\mathrm{i}}^{\infty}$ the space of all inner functions in $H^{\infty}$. If $m \in H_{\mathrm{i}}^{\infty}$, then $P(m)$ denotes the orthogonal projection from $H^{2}$ onto the space $H(m)=\left(m H^{2}\right)^{\perp}=H^{2} \ominus m H^{2}$ and

$$
S(m) u=P_{m}(u \cdot m), \quad \text { for every } u \in H(m) \text {. }
$$


$S(m)$ is a contraction of class $C_{0}$ whose minimal function is $m$. For a contraction $T \in C_{0}$ with minimal function $m$ there exists a unique sequence of inner functions $m_{1}=m, m_{2}, m_{3}, \ldots$ such that $m_{i+1}$ divides $m_{i}$ for every $i$ and the Jordan model of $T$ is

$$
S\left(m_{1}\right) \oplus S\left(m_{2}\right) \oplus \ldots \quad \text { on } H\left(m_{1}\right) \oplus H\left(m_{2}\right) \oplus \ldots
$$

It was shown in [3] that a $C_{0}$ contraction $T$ has the property $(\mathrm{P})$ if and only if the greatest common inner divisor of the functions $m_{i}$ in the Jordan model of $T$ is 1 .

In [18] a similar characterization of a.c. $C_{11}$ contractions $T$ having the property (P) was given. It is well known that $T$ is quasisimilar to an a.c. unitary operator $U$. According to the theory of spectral multiplicities there exist a sequence $E_{1}, E_{2}, \ldots$ of measurable subsets of the unit circle $\mathbb{T}$ such that $T$ is quasisimilar to

$$
M\left(E_{1}\right) \oplus M\left(E_{2}\right) \oplus \ldots \quad \text { on } L^{2}\left(E_{1}\right) \oplus L^{2}\left(E_{2}\right) \oplus \ldots
$$

For $E \subset \mathbb{T}, L^{2}(E)$ denotes the space of all functions from $L^{2}(\mathbb{T})$ that vanish outside $E$ and $M(E)$ denotes the operator of multiplication by $e^{i t}$, i.e. the restriction of the usual bilateral shift to its reducing subspace $L^{2}(E)$.

According to [18, Theorem 1], $T$ has the property $(\mathrm{P})$ if and only if the Lebesgue measure of $\bigcap_{n>1} E_{n}$ is zero. According to Lemma 3 of [39] for every a.c. unitary operator $U$ there exists a c.n.u. weak contraction similar to $U$. Therefore not every weak contraction of class $C_{11}$ has the property $(\mathrm{P})$ (while all weak $C_{0}$ contractions have $\left.(\mathrm{P})\right)$.

Problem. For $C_{0}$ contractions, $(\mathrm{P})$ implies $(\mathrm{L})$. It is not known whether this implication holds for $C_{11}$ contractions.

R e marks. 1. The problem of reflexivity and hyperreflexivity of a $C_{0}$ contraction was reduced to the problem of reflexivity of a single Jordan block $S(m)$ in [5]. Recently, Kapustin [16] has characterized those inner functions $m$ for which $S(m)$ is reflexive.

2. The property (L) was first proved for contractions with finite defect indices (see [28], [30], [32], [33]). Then these results were generalized to some contractions with infinite defect indices, in particular to all weak contractions (see [35]-[37], [39]).

3. In [16] and [17] a relation called pseudosimilarity was defined. It is stronger than quasisimilarity and preserves many of the properties of invariant subspace lattices and operator algebras connected with an operator $T$. Most of the above mentioned results were first proved for the Jordan models and the second step was to show that in those particular cases quasisimilarity preserves the properties in question. The reason was that in most cases the Jordan model of $T$ was pseudosimilar to $T$.

4. In [1] some nilpotent operators in Banach space were proved to have the property $(\mathrm{L})$. 
4. Hyperinvariant subspaces of isometries. Let $T \in \mathcal{B}(H)$ be an isometry. The hyperinvariant subspaces of $T$ were described in [11] in terms of the canonical decomposition (III). Using this decomposition the characterization of isometries having the property (L) and of hyperreflexive isometries were obtained in [36], [41]. Hyperreflexive isometries were characterized much earlier by V. S. Shul'man [23]. The following theorem puts together the above mentioned results.

Theorem 4. Consider the canonical decomposition (III) of an isometry $T \in$ $\mathcal{B}(H)$. Then:

(1) $L \in \operatorname{Lat}\{T\}^{\prime}$ if and only if $L=L_{1} \oplus L_{2} \oplus L_{3}$, where $L_{i} \in \operatorname{Lat}\left\{T_{i}\right\}^{\prime}$ for $i=1,2,3$, and either $L_{1}=\{0\}$ or $L_{2}=H_{2}$.

(2) Every $A \in\{T\}^{\prime}$ has a matrix form

$$
A=\left(\begin{array}{ccc}
A_{1} & 0 & 0 \\
X & A_{2} & 0 \\
0 & 0 & A_{3}
\end{array}\right),
$$

where $A_{i} \in\left\{T_{i}\right\}^{\prime}, i=1,2,3$, and $X T_{1}=T_{2} X$.

(3) $A \in \operatorname{Alg} \operatorname{Lat}\{T\}^{\prime}$ if and only if it has the above matrix form with some $X \in \mathcal{B}\left(H_{1}, H_{2}\right)$.

(4) $T$ is hyperreflexive if and only if either $T_{1}=0$ or $T_{2}=0$.

(5) $T$ has the property $(L)$ if and only if it is hyperreflexive.

R e m a r k. It was shown in [41] that neither implication in (5) holds for general bounded operators in Hilbert space.

Every isometry is reflexive [9] and so for isometries as well as for operators in finite-dimensional spaces and for $C_{0}$ contractions [5] hyperreflexivity implies reflexivity. It has been an open problem whether there exists a hyperreflexive operator which is not reflexive. We give a solution of this problem in the following section.

5. Hyperreflexivity and reflexivity. We start with a simple sufficient condition for hyperreflexivity:

TheOREM 5. Let $T \in \mathcal{B}(H)$. If the closed linear span of all eigenvectors of $T$ is $H$, then $T$ is hyperreflexive.

Proof. If $\lambda$ is an eigenvalue of $T$, then $\operatorname{ker}(\lambda-T)$ is hyperinvariant for $T$. It follows that for every $A \in \operatorname{Alg} \operatorname{Lat}\{T\}^{\prime}$ and for every eigenvector $h \in \operatorname{ker}(\lambda-T)$,

$$
A T h=A(\lambda h)=\lambda A h=T A h .
$$

Since the eigenvectors span $H$, we have $A T=T A$, i.e. $T$ is hyperreflexive.

Even the very strong assumptions of the preceding theorem are not sufficient for $T$ to be reflexive. Before showing this fact we prove another simple result. 
TheOrem 6. Let $T \in \mathcal{B}(H)$, let $\lambda$ be an eigenvalue of $T$ and let $A \in \operatorname{Alg} \operatorname{Lat} T$. Then there exists a complex number a $(\lambda)$ such that every $h \in \operatorname{ker}(\lambda-T)$ is an eigenvector of $A$ with eigenvalue $a(\lambda)$. Consequently, if in addition the eigenvectors of $T$ span $H$, then $A \in\{T\}^{\prime \prime}$.

Pr o of. The 1-dimensional space spanned by $h \in \operatorname{ker}(\lambda-T)$ is invariant for $T$ and so also for $A$. Therefore there exists a complex number $a$ such that $A h=a h$. If $g \in \operatorname{ker}(\lambda-T)$ is another eigenvector, then there are $b, c \in \mathbb{C}$ such that $A g=b g$ and $A(h+g)=c(h+g)=a h+b g$. It is easy to prove that $b=c=a$.

Recently, Larson and Wogen [19] have constructed a reflexive operator $T \in$ $\mathcal{B}(H)$ whose eigenvectors span $H$ such that the operator $T \oplus 0 \in \mathcal{B}(H \oplus K)$ with $\operatorname{dim} K \geq 1$ is not reflexive. They used this example to give solutions to several open problems. It turns out that the same example also answers (negatively) the problem whether every hyperreflexive operator must be reflexive. Indeed, the eigenvectors of $T \oplus 0$ span $H \oplus K$ and so $T \oplus 0$ is hyperreflexive.

Problems. 1. If a nilpotent operator $T$ is hyperreflexive, then it is equal to 0 [12]. Does there exist a non-zero hyperreflexive operator in a (necessarily infinite-dimensional) Hilbert space with spectrum $\sigma(T)=\{0\}$ ?

2. Every contraction of class $C_{11}$ is hyperreflexive because it is quasisimilar to a unitary operator and quasisimilarity preserves hyperreflexivity. Does there exist a non-reflexive contraction of class $C_{11}$ ? Note that since every $C_{11}$ contraction $T$ has reflexive bi-commutant [27], we have the implication $A \in \operatorname{Alg} \operatorname{Lat} T \Rightarrow A \in\{T\}^{\prime \prime}$.

\section{References}

[1] M. Barraa, Sous-espaces hyperinvariants d'un opérateur nilpotent sur un espace de Banach, J. Operator Theory 21 (1989), 315-321.

[2] M. Benlarbi Delaï et B. Charles, Description de AlgLat A pour un opérateur A algébrique, Linear Algebra Appl. 187 (1993), 105-108.

[3] H. Bercovici, $C_{0}$-Fredholm operators II, Acta Sci. Math. (Szeged) 42 (1980), 3-42.

[4] - Operator Theory and Arithmetic in $H^{\infty}$, Math. Surveys Monographs 26, Providence, R.I., 1988.

[5] H. Bercovici, C. Foiaş and B. Sz.-Nagy, Reflexive and hyper-reflexive operators of class $C_{0}$, Acta Sci. Math. (Szeged) 43 (1981), 5-13.

[6] H. Bercovici and L. Kérchy, Quasisimilarity and properties of the commutant of $C_{11}$ contractions, ibid. 45 (1983), 67-74.

[7] L. Brickman and P. A. Fillmore, The invariant subspace lattice of a linear transformation, Canad. J. Math. 19 (1967), 810-822.

[8] J. B. Conway and P. Y. Wu, The splitting of $Q\left(T_{1} \oplus T_{2}\right)$ and related questions, Indiana Univ. Math. J. 26 (1977), 41-56.

[9] J. A. Deddens, Every isometry is reflexive, Proc. Amer. Math. Soc. 28 (1971), 509-512.

[10] J. A. Deddens and P. A. Fillmore, Reflexive linear transformations, Linear Algebra Appl. 10 (1975), 89-93.

[11] R. G. Douglas, On the hyperinvariant subspaces for isometries, Math. Z. 107 (1968), $297-300$. 
[12] Š. Drahovský and M. Zajac, Hyperreflexive operators on finite dimensional Hilbert spaces, Math. Bohem. 118 (1993), 249-254.

[13] P. A. Fillmore, D. A. Herrero and W. F. Longstaff, The hyperinvariant subspace lattice of a linear transformation, Linear Algebra Appl. 17 (1977), 125-132.

[14] P. R. Halmos, Eigenvectors and adjoints, ibid. 4 (1971), 11-15.

[15] R. A. Horn and C. R. Johnson, Topics in Matrix Analysis, Cambridge University Press, 1991.

[16] V. V. Kapustin, Reflexivity of operators: general methods and a criterion for almost isometric contractions, Algebra i Analiz 4 (2) (1992), 141-160 (in Russian); English transl.: St. Petersburg Math. J. 4 (1993), 319-335.

[17] V. V. Kapustin and A. V. Lipin, Operator algebras and invariant subspace lattices. I, Zap. Nauchn. Sem. Leningrad. Otdel. Mat. Inst. Steklov. (LOMI) 178 (1989), 23-56 (in Russian).

[18] L. Kérchy, On the commutant of $C_{11}$-contractions, Acta Sci. Math. (Szeged) 43 (1981), $15-26$.

[19] D. R. Larson and W. R. Wogen, Reflexivity properties of $T \oplus 0$, J. Funct. Anal. 92 (1990), 448-467.

[20] S.-C. Ong, Remarks on invariant subspaces of finite dimensional operators, Linear Algebra Appl. 42 (1982), 99-101

[21] -, What kind of operators have few invariant subspaces?, ibid. 95 (1987), 181-185.

[22] —, On equality of few invariant subspace lattices of operators, ibid. 144 (1991), 23-27.

[23] V. S. Shul'man, The Fuglede-Putnam theorem and reflexivity, Dokl. Akad. Nauk SSSR 210 (1973), 543-544 (in Russian); English transl.: Soviet Math. Dokl. 14 (1973), 784-786.

[24] S. O. Sickler, The invariant subspaces of almost unitary operators, Indiana Univ. Math. J. 24 (1975), 636-649.

[25] D. A. Suprunenko and R. I. Tyshkevich, Commutative Matrices, Nauka i Tekhnika, Minsk, 1966 (in Russian); English transl.: Academic Press, New York, 1968.

[26] B. Sz.-Nagy and C. Foias, Harmonic Analysis of Operators on Hilbert Space, NorthHolland, Amsterdam, and Akadémiai Kiadó, Budapest, 1970.

[27] K. Takahashi, Double commutants of operators quasi-similar to normal operators, Proc. Amer. Math. Soc. 92 (1984), 404-406.

[28] M. Uchiyama, Hyperinvariant subspaces of operators of class $C_{0}(N)$, Acta Sci. Math. (Szeged) 39 (1977), 179-184.

[29] -, Hyperinvariant subspaces for contractions of class C.0. Hokkaido Math. J. 6 (1977), 260-272.

[30] P. Y. Wu, The hyperinvariant subspace lattice of the contraction of class $C_{.0}$, Proc. Amer. Math. Soc. 72 (1978), 527-530.

[31] -, Hyperinvariant subspaces of $C_{11}$ contractions, ibid. 75 (1979), 53-58.

[32] - , Hyperinvariant subspaces of $C_{11}$ contractions, II, Indiana Univ. Math. J. 27 (1978), 805-812.

[33] - , Hyperinvariant subspaces of weak contractions, Acta Sci. Math. (Szeged) 41 (1979), 259-266.

[34] -, Which linear transformations have isomorphic hyperinvariant subspace lattices?, Linear Algebra Appl. 169 (1992), 163-178.

[35] M. Zajac, Hyperinvariant subspace lattice of some $C_{0}$-contractions, Math. Slovaca 31 (1981), 397-404.

[36] - Hyperinvariant subspace lattice of weak contractions, ibid. 33 (1983), 75-80.

[37] - Hyperinvariant subspaces of weak contractions, in: Oper. Theory: Adv. Appl. 14, Birkhäuser, 1984, 291-299.

[38] —, Hyperinvariant subspace lattice of isometries, Math. Slovaca 37 (1987), 291-297. 
[39] M. Zajac, Hyperinvariant subspaces of weak contractions, II, in: Oper. Theory: Adv. Appl. 28, Birkhäuser, 1988, 317-322.

[40] - On the singular unitary part of a contraction, Rev. Roumaine Math. Pures Appl. 35 (1990), 379-384.

[41] - , Hyper-reflexivity of isometries and weak contractions, J. Operator Theory 25 (1991), $43-51$. 